\title{
A New High-Order Alternating Group Iterative Scheme
}

\author{
Y.M. Shi \\ School of Mathematic and Quantitative Economics \\ Shandong University of Finance and Economics \\ Jinan, China
}

\begin{abstract}
In this paper, by constructing a new high-order parallel alternating group iterative algorithm, we solve the two point boundary value problem. The main method is to dividing the system of different equations into a set of subsystem which can be solved individually in parallel and sixth order accurate. We have obtained the main results and given some examples to validate our results in addition. It shows that the analysis is correct and the algorithm is feasible and efficient.
\end{abstract}

Keywords-two point boundary value problem; sixth order; alternating group iterative scheme; convergence

\section{INTRODUCTION}

The solution for the two-point boundary value problems is one of the most classic problems in the numerical approximation of differential equations. By developing the alternating method, in [1-3], D. J. Evans and A. R. B. Abdullah had proposed the Alternating Group Explicit (AGE) strategy to solve the differential equations. D. J. Evans [4] also solved large linear systems by using iterative methods. This scheme is capable of parallel computation; however, its truncation error is only second order or lower. In [5], a fourth order Alternating Group Iterative (AGI) algorithm was constroucted for two point boundary value problem. Thus we propose to derive a new alternating group iterative method, which has truncation error of sixth order.

\section{DIFFERENCE SCHEME}

We consider the periodic solution for the following second order self-adjoint ordinary differential equation (ODE):

$$
\begin{gathered}
L u=-\frac{d^{2} u}{d x^{2}}+q u=f, \quad a<x<b, \\
u(a)=u(a+H)
\end{gathered}
$$

Here $q, f$ are given real continuous functions on $a \leq x \leq b$ and $H=b-a$ represents the length of one period, with $q \geq 0$.

We partition the interval $[a, b]$ into subintervals of equal length $h=H / J$ and let the grid points be $x_{i}=a+(i-1) h, i=1, \cdots, J+1 . u_{i}, q_{i}, f_{i}$ denote the finite difference approximations of $u\left(x_{i}\right), q\left(x_{i}\right), f\left(x_{i}\right)$ respectively. The parallel finite difference scheme is constructed as follows:
$L_{h} u_{i}=\frac{-2 u_{i+3}+27 u_{i+2}-270 u_{i+1}+490 u_{i}-270 u_{i-1}+27 u_{i-2}-2 u_{i-3}}{180 h^{2}}+q_{i} u_{i}=f_{i}$.

or

$-\frac{2 r}{180} u_{i+3}+\frac{27 r}{180} u_{i+2}-\frac{270 r}{180} u_{i+1}+\left(\frac{490 r}{180}+q_{i}\right) u_{i}-\frac{270 r}{180} u_{i-1}+\frac{27 r}{180} u_{i-2}-\frac{2 r}{180} u_{i-3}=f_{i}$

Where $r=\frac{1}{h^{2}}, i=1,2, \cdots, J$.The truncation error at point $x=x_{i}$ for (3) is:

$$
R_{i}=-\frac{h^{6}}{560}\left[\frac{d^{8} u}{d x^{8}}\right]_{i}+o\left(h^{7}\right)
$$

We can rewrite the linear difference (3) in matrix form

$$
A u=F
$$

where
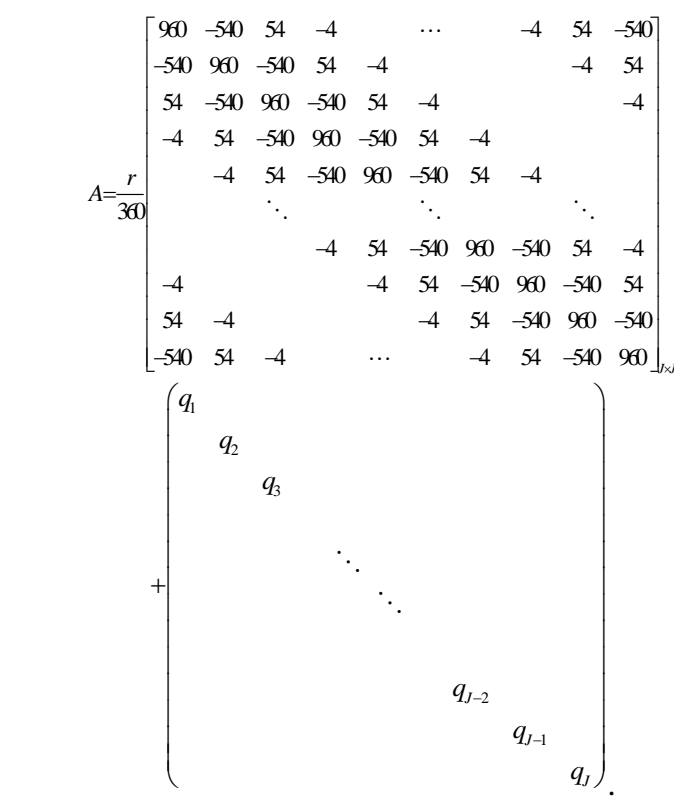

$u=\left(u_{1}, u_{2}, \cdots, u_{J}\right)^{T}, F=\left(f_{1}, f_{2}, \cdots, f_{J-2}, f_{J-1}, f_{J}\right)^{T}$. 
We assume that $J=6 k$, and splitting the matrix $A$ into the sum of three matrices

$$
A=G_{1}+G_{2}+\sum
$$

where

$$
G_{1}=\frac{r}{360}\left[\begin{array}{cccc}
Q_{3} & & & \\
& P_{9}^{(1)} & & \\
& \ddots & & \\
& & Q_{3} & \\
& & & P_{9}^{(k)}
\end{array}\right], \Sigma=\left[\begin{array}{cccc}
q_{1} & & & \\
& q_{2} & & \\
& \ddots & & \\
& & q_{J-1} & \\
& & & q_{J}
\end{array}\right] \text {. }
$$

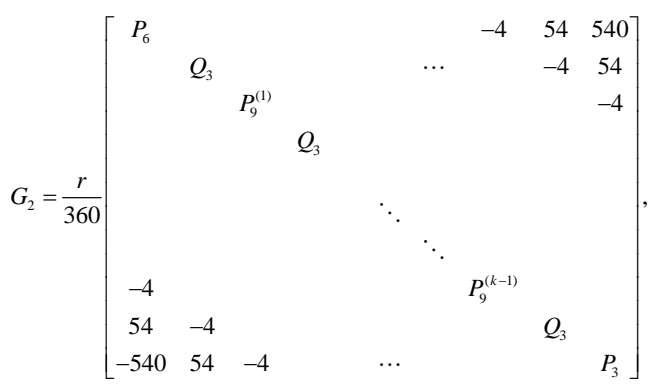

in which

$$
P_{9}^{(i)}=\left(\begin{array}{cccccccccc}
247 & -270 & 27 & -4 & & & & & \\
-270 & 490 & -270 & 54 & -4 & & & & \\
27 & -270 & 733 & -540 & 54 & -4 & & & \\
-4 & 54 & -540 & 980 & -540 & 54 & -4 & & \\
& -4 & 54 & -540 & 980 & -540 & 54 & -4 & \\
& & -4 & 54 & -540 & 980 & -540 & 54 & -4 \\
& & -4 & 54 & -540 & 733 & -270 & 27 \\
& & & -4 & 54 & -270 & 490 & -270 \\
& & & & -4 & 54 & -270 & 247
\end{array}\right),
$$$$
\begin{gathered}
P_{6}=\left(\begin{array}{cccccc}
980 & -540 & 54 & -4 & & \\
-540 & 980 & -540 & 54 & -4 & \\
54 & -540 & 980 & -540 & 54 & -4 \\
-4 & 54 & -540 & 733 & -270 & 27 \\
& -4 & 54 & -270 & 490 & -270 \\
& -4 & 54 & -270 & 247
\end{array}\right) P_{3}=\left(\begin{array}{ccc}
247 & -270 & 27 \\
-270 & 490 & -270 \\
27 & -270 & 733
\end{array}\right) \\
Q_{3}=\left(\begin{array}{lll}
0 & 0 & 0 \\
0 & 0 & 0 \\
0 & 0 & 0
\end{array}\right)
\end{gathered}
$$

By using (6) and (7), we have

$$
\left(G_{1}+G_{2}+\Sigma\right) u=F
$$

and let us consider two equivalent forms,

$$
\left\{\begin{array}{l}
\left(\rho I+\bar{G}_{1}\right) \tilde{u}^{(k+1)}=\left[\left(\rho I-\bar{G}_{2}\right) u^{(k)}+F\right] \\
\left(\rho I+\bar{G}_{2}\right) u^{(k+1)}=\left[\left(\rho I-\bar{G}_{1}\right) \tilde{u}^{(k+1)}+F\right] k=0,1,2, \cdots,
\end{array}\right.
$$

where $\bar{G}_{1}=G_{1}+\frac{1}{2} \sum, \bar{G}_{2}=G_{2}+\frac{1}{2} \sum, \quad \rho$ is a positive iteration parameter and

$$
\left(\rho I+\bar{G}_{1}\right)^{-1},\left(\rho I+\bar{G}_{2}\right)^{-1} \text { exist. }
$$

\section{THE CONVERGENCE ANALYSIS}

Lemma 1 If $A=\left(a_{i j}\right) \in C^{m \times n}$, then $\|A\|_{2}=\sqrt{\lambda_{1}}, \quad \lambda_{1}$ is the max eigenvalue of $A^{H} A$.

According to lemma 1 , if $A$ is real and symmetric matrix, then $\|A\|_{2}=\rho(A), \rho(A)$ is the spectral radius of $A$.

Lemma 2 In (8), $\bar{G}_{1}$ and $\bar{G}_{2}$ are both positive definite matrices. Eliminating $\tilde{u}^{(k+1)}$ of (9), we have

$$
u^{(k+1)}=T u^{(k)}+\tilde{F} .
$$

here

$$
\begin{aligned}
& T=\left(\rho I+\bar{G}_{2}\right)^{-1}\left(\rho I-\bar{G}_{1}\right)\left(\rho I+\bar{G}_{1}\right)^{-1}\left(\rho I-\bar{G}_{2}\right) \\
& \tilde{F}=\left(\rho I+\bar{G}_{2}\right)^{-1}\left[\left(\rho I-\bar{G}_{1}\right)\left(\rho I+\bar{G}_{1}\right)^{-1} F+F\right] .
\end{aligned}
$$

Now define the matrix:

$$
\tilde{T}=\left(\rho I+\bar{G}_{2}\right) T\left(\rho I+\bar{G}_{2}\right)^{-1},
$$

which is similar to $T$ and thus has the same eigenvalues as $T$.Then it is evident that:

$$
\begin{gathered}
\rho(T)=\rho(\tilde{T})=\|\tilde{T}\|_{2} \leq \\
\left\|\left(\rho I-\bar{G}_{1}\right)\left(\rho I+\bar{G}_{1}\right)^{-1}\right\|_{2} \times\left\|\left(\rho I-\bar{G}_{2}\right)\left(\rho I+\bar{G}_{2}\right)^{-1}\right\|_{2} \\
=\max _{i}\left|\frac{\rho-\mu_{i}}{\rho+\mu_{i}}\right| \cdot \max _{i}\left|\frac{\rho-\eta_{i}}{\rho+\eta_{i}}\right| .
\end{gathered}
$$

Here $\mu_{i}, \eta_{i}(i=1,2, \cdots, J)$ are respectively the eigenvalues of $\bar{G}_{1}$ and $\bar{G}_{2}$.According to the lemmas, $\bar{G}_{1}$ and $\bar{G}_{2}$ are positive definite matrices, therefore $\mu_{i}>0, \eta_{i}>0(i=1,2, \cdots, J)$ and we have $\rho(T)<1$. Hence the convergence of the alternating iterative scheme is proved.

\section{NUMERICAL EXPERIMENT}

We perform the numerical simulations using the following model problem:

$$
\left\{\begin{array}{l}
-\frac{d^{2} u}{d x^{2}}+x u=\sin x(x+1) \quad 0<x<2 \pi, \\
u(0)=u(2 \pi) .
\end{array}\right.
$$


The exact solution is:

$$
u=\sin x
$$

The error bounds of the sixth order scheme are examined first. The $L^{2}$ errors are defined to be $e_{h}=\|u-u(x)\|_{L^{2}}$, and are computed based on the following four different $\rho$, and four different $h$ :

$$
\rho=1,3,5,7 ; h=\frac{2 \pi}{12}, \frac{2 \pi}{30}, \frac{2 \pi}{60}, \frac{2 \pi}{120} .
$$

The results are listed in Table 1 .It is not hard to see that the $L^{2}$ errors of the six-order scheme are order 6 in space, which comfirms the earlier results in Section 2.

Next, we compare the accuracy of the sixth order AGI scheme to the fourth order AGI scheme. The computation is based on the same $\rho$, and $h$ is taken to be $\pi / 12$.The absolute errors(ae) and the percentage errors(pe) for these two schemes are listed in Table 2 and plotted in Figure 1 and Figure 2 at the end of paper. Evidently, the results show that the sixth order AGI scheme is more accurate than the fourth order AGI scheme .

TABLE I. CONVERGENCE RATES FOR THE SIXTH ORDER SCHEME

\begin{tabular}{|c|c|c|c|c|c|c|c|c|}
\hline \multirow{2}{*}{$J$} & \multicolumn{2}{|c|}{$\rho=1$} & \multicolumn{2}{c|}{$\rho=3$} & \multicolumn{2}{c|}{$\rho=5$} & \multicolumn{2}{c|}{$\rho=7$} \\
\cline { 2 - 9 } & $e_{h} * 10^{5}$ & $e_{h} / h^{6}$ & $e_{h} * 10^{7}$ & $e_{h} / h^{6}$ & $e_{h} * 10^{7}$ & $e_{h} / h^{6}$ & $e_{h} * 10^{7}$ & $e_{h} / h^{6}$ \\
\hline 12 & 3.012 & 0.001 & 301 & 0.001 & 301 & 0.001 & 301 & 0.001 \\
\hline 30 & 0.020 & 0.002 & 1.98 & 0.002 & 1.98 & 0.002 & 1.98 & 0.002 \\
\hline 60 & 1.579 & 11.98 & 0.044 & 0.003 & 0.044 & 0.003 & 0.44 & 0.003 \\
\hline 120 & 146 & 1463 & 0.017 & 8.21 & 0.001 & 0.005 & $\begin{array}{c}0.0009 \\
6\end{array}$ & 0.005 \\
\hline
\end{tabular}

\begin{tabular}{|c|c|c|c|c|c|c|}
\hline & \multicolumn{6}{|c|}{$J=24, \Delta x=\pi / 12, \rho=2$} \\
\hline & $x=0.2 \epsilon$ & $x=1.05$ & $x=2.10$ & $x=3.4 C$ & $x=4.2 C$ & $x=6.02$ \\
\hline $\begin{array}{l}\text { 6th- } \\
\text { order } \\
\text { AGI }\end{array}$ & & & & & & \\
\hline ae & $.13310^{6}$ & $.244 \times 10^{-6}$ & $.187 \times 10^{6}$ & $.17410^{7}$ & $.890 \times 10^{-7}$ & $.190 \times 10^{7}$ \\
\hline pe & $.51 \times 10^{6}$ & $.282 \times 10^{6}$ & $.216 \times 10^{6}$ & $.67310^{7}$ & $.103 \times 10^{6}$ & $.732 \times 10^{7}$ \\
\hline $\begin{array}{c}\text { 4th- } \\
\text { order } \\
\text { AGI }\end{array}$ & & & & & & \\
\hline ae & $.12 \approx 10^{4}$ & $.22 z 10^{4}$ & $.17010^{4}$ & $.159 \times 10^{5}$ & $.81 \mathrm{k} 10^{5}$ & $.17 \mathrm{\gtrless} 10^{5}$ \\
\hline pe & $.47 \mathrm{k} 10^{4}$ & $.25610^{4}$ & $.196 \times 10^{4}$ & $.61410^{4}$ & $.93610^{5}$ & $.66 * 10^{5}$ \\
\hline $\begin{array}{c}\text { Exact } \\
\text { solution }\end{array}$ & .259 & .866 & .866 & -.259 & -.866 & -.259 \\
\hline
\end{tabular}

TABLE II. ABSOLUTE AND PERCENTAGE ERRORS

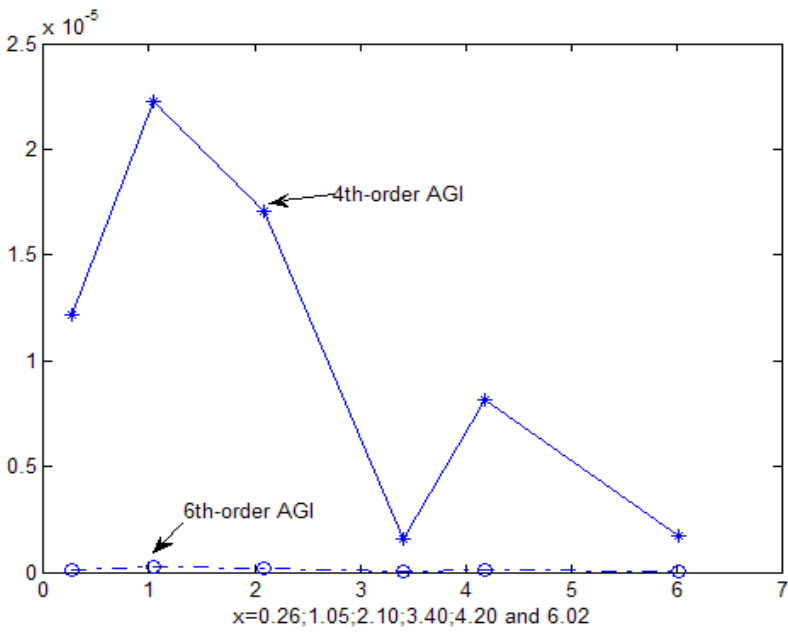

FIGURE I. ABSOLUET ERRORS $J=24, \rho=2$

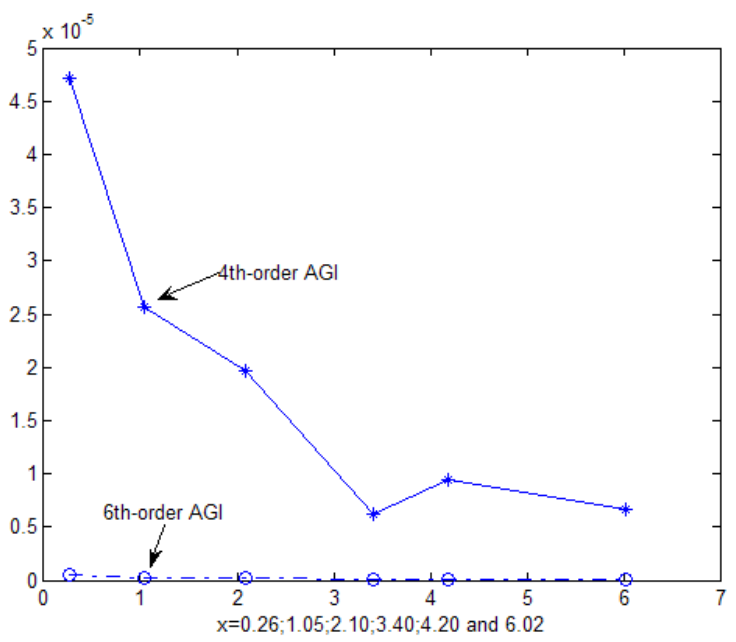

FIGURE II. PERCENTAGE ERRORS $J=24, \rho=2$

\section{CONCLUSIONS}

In this paper, a sixth order alternating group iterative algorithm is derived for two point boundary value problem. The scheme has truncation error of sixth order in space which is higher than similar fourth order AGI scheme. The scheme is proved to be stable under reasonable condition. Numerical example is also prensented.

\section{ACKNOWLEDGEMENT}

This work is supported by NSF of China (11326226).

\section{REFERENCES}

[1] D.J. Evans and A.R.B. Abdullah, Group Explicit Methods for Parabolic Equations. International Journal of Computer Mathematics, Vol.14, pp.73-105, 1983.

[2] D.J. Evans and A.R.B. Abdullah. A New Explicit Method for the Diffusion-Convection Equation. Computers \& Mathematics with Application, Vol.11, pp.145-154, 1985.

[3] D.J. Evans. Alternating Group Explicit (AGE) Iterative Methods. International Journal of Computer Mathematics, Vol.19, pp.309-326, 1986. 
[4] D.J. Evans. Group Explicit Iterative Methods for Solving Large Linear Systems. International Journal of Computer Mathematics, Vol.17, pp.81-108, 1985.

[5] Yumin Shi,A high-order alternating group iterative algorithm,2011 Fourth International Joint Conference on Computational Sciences and Optimization,pp.12-15,2011

[6] Dayi Yi and Daoqi Chen. Introduction to Numerical Analysis.Zhejiang University Press,2000. 